\title{
The Pulsed Glow Discharge as an Elemental Ion Source
}

\author{
J. A. Klingler, P. J. Savickas, ${ }^{*}$ and W. W. Harrison \\ Department of Chemistry, University of Florida, Gainesville, Florida, USA
}

\begin{abstract}
A pulsed glow discharge, rather than a conventional constant dc voltage discharge, is used as an ion source for a quadrupole mass spectrometer. Both sputter yield and ion signal are enhanced by using the pulsed system because of an increase in the voltage necessary to maintain a constant average current at the cathode over the pulse period. Irregularities are seen in the pulse spectrum that appear as rapid surges in the ion signal for both sputtered and contaminant gas species. These peaks appear at the beginning of the pulse for gaseous species but are limited to the postpulse period for sputtered species. Differences in the signal forms allow for the discrimination against selected types of ion signals by using narrow data collection gates placed over different portions of the pulse period. (J Am Soc Mass Spectrom 1990, 1, 138-143)
\end{abstract}

$\mathrm{G}$ low discharge mass spectrometry (GDMS) is finding increasing popularity for the elemental analysis of solids. Its advantages include detection limits of parts per billion, minimal matrix effects, stable ion signals, simple sample preparation, and isotopic information. The glow discharge is normally formed by a dc discharge in a low-pressure argon environment. Ionized argon ions are accelerated onto the sample cathode, which sputters off analyte atoms that are subsequently ionized in the plasma by electron ionization (EI) or Penning ionization steps [1].

The intensity of the ion signal is normally proportional to the sputtered atom density [2]. An increase in the sputter yield can be effected by raising the discharge voltage, thereby increasing the average energy of the bombarding ions. Higher voltage discharges should result in larger ion signals and enhanced detection limits. In the abnormal glow discharge [3], however, the voltage is tied to the current, and both increase together at constant pressure. Operation in the $\mathrm{dc}$ mode at high voltages can lead to overheating of the cathodes, unwanted thermal emissions, and unstable discharges. Cooling of the sample is one option to alleviate the heating problem, but water cooling systems introduce additional problems in vacuum environments and, in any case, are not very efficient for cooling the pin-shaped sample electrodes normally used in GDMS.

Pulsing the glow discharge permits operation at higher discharge voltages compared to the comparable $\mathrm{dc}$ mode at the same average current. If the average current density in a pulsed glow discharge is main-

\footnotetext{
* Current address: Dow Chemical, Midland, Michigan.

Address reprint requests to $W$. W. Harrison, Department of Chemistry, University of Florida, Gainesville, FL 32611.
}

tained constant and the duty cycle of the pulse period is reduced, then the voltage at the cathode must be higher to maintain the current. Cathodic heating generated during the "on" portion of the duty cycle tends to dissipate during the "off" portion. Variation of the duty cycle allows the analyst to adjust discharge voltage, sputter yield, and ion signal, within power supply constraints.

Pulsed hollow cathode lamps showed emission intensity increases of several hundredfold [4] with no increase in line width. Similar sources are used in atomic fluorescence $[5,6]$. Time-resolved emission line profiles were examined in pulsed hollow cathode lamps [7]. The effect noted in emission signals led us to investigate the possibility that similar advantages might be observed in elemental ion intensities.

This study concerns the use of a pulsed glow discharge ion source in GDMS and compares its operation with the conventional dc approach. During the course of our investigations, certain time-dependent anomalies were observed in the ion signal. These timeresolved ion variations at different segments of the pulse are described, including a potentially advantageous increase in analytical signal immediately after cessation of the pulse. A method is reported that utilizes the time-dependent ion signals to discriminate against various interference species in the glow discharge.

\section{Experimental}

The mass spectrometer system used in these experiments is described elsewhere [8]. The peripheral equipment driving the discharge and the timing of the data collection system are described here.

Control of the plasma and the data collection system 


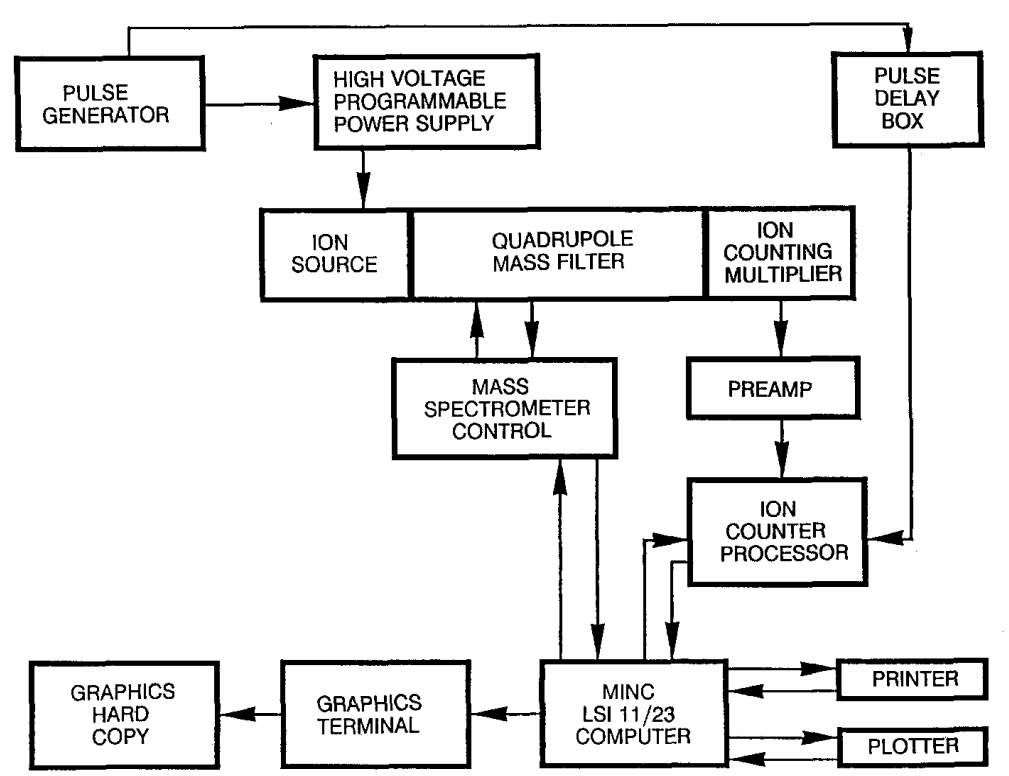

Figure 1. Block diagram of the components for pulsed GDMS. centers around a Hewlett-Packard 8003A pulse generator (see Figure 1). The square-wave generator allows the operator to control the pulse signal amplitude, frequency, and duty cycle. This variable pulse signal is used to drive a Kepco OPS3500 operational power supply that can closely follow the pulse signal waveform.

The pulse generator also provides a $5-\mathrm{V}$ pulse synchronized with the low-to-high portion of the controlling square-wave signal. This timing signal is led into a pulse delay box and is then passed to a signal processor (Princeton Applied Research Model 1112). Ion signals from the mass spectrometer are collected through data gates that are opened by the synchronization pulse; the duration of these gates is controlled by the signal processor. By using these gates, a data acquisition window from $1 \times 10^{-6} \mathrm{~s}$ up to the entire pulse period is possible. The data acquisition window can be positioned over any portion of the pulse cycle by delaying the arrival of the synchronization pulse relative to the application of the high voltage.

Voltage and current to the discharge are monitored by D'Arsonval deflection meters. The pulse voltage and current as indicated by the meters are values averaged out over the period of the on/off cycle of the pulse. Hence a reading of $2 \mathrm{~mA}$ on the current meter at a $50 \%$ duty cycle corresponds to $4 \mathrm{~mA}$ current arriving at the cathode during the "on" portion of the duty cycle. To maintain an average current of $2 \mathrm{~mA}$ at a $25 \%$ duty cycle, the "on" current must be $8 \mathrm{~mA}$, with a corresponding increase in the voltage.

Samples were prepared from SRM 1200 series low alloy steel, SRM C1100 series brass, and high purity copper (Alfa). Specimens were cut into pins $2 \mathrm{~mm}$ in diameter with $5 \mathrm{~mm}$ exposed to the discharge. Each pin was polished with $5 \mu \mathrm{m}$ alumina and then rinsed with solvent. Special precautions were taken with specimens used in weight loss studies. These pins were $1.59 \mathrm{~mm}$ in diameter, and their surfaces were finished by using the automatic carriage feed of a precision metal lathe without cutting oils. The pins were cleaned in an ultrasonic bath with soapy water followed sequentially by distilled water, ethanol, and finally acetone. A Sartorius microanalytical balance (sensitive to $1 \mu \mathrm{g}$ ) was used for specimen weighing after each sputter period. The discharge gas in all experiments was reagent grade argon.

\section{Results and Discussion}

The efficacy of a pulsed glow discharge as an ion source can be explored in several ways. An enhanced pulse effect should manifest itself in a larger sputtered atom population, complementary to a larger ion signal. Sputter yield has been shown to increase with ion energy up to the low kilovolt region [9]. The average ion energies in a glow discharge are much lower than the power supply discharge voltage owing to chargeexchange reactions occurring in the dark space [10]. Average energies may be about $25-30 \%$ of the applied voltage, leading to $200-400-\mathrm{eV}$ sputter ions for our typical dc discharge. In the pulsed mode, the significant voltage increase during the "on" cycle should create a larger sputter yield of target atoms.

To determine the extent to which a pulsed discharge may sputter more material than a dc discharge, a weight loss comparison was performed. The pulsed discharge was operated at $50 \mathrm{~Hz}$ with a $50 \%$ duty cycle. Both the dc and pulse discharges were maintained at 1.0 torr argon with an average current of $1 \mathrm{~mA}$. Generally, at a $50 \%$ duty cycle, the applied voltage for a pulsed discharge must be increased to $40-60 \%$ above the $\mathrm{dc}$ applied voltage to maintain a constant average 


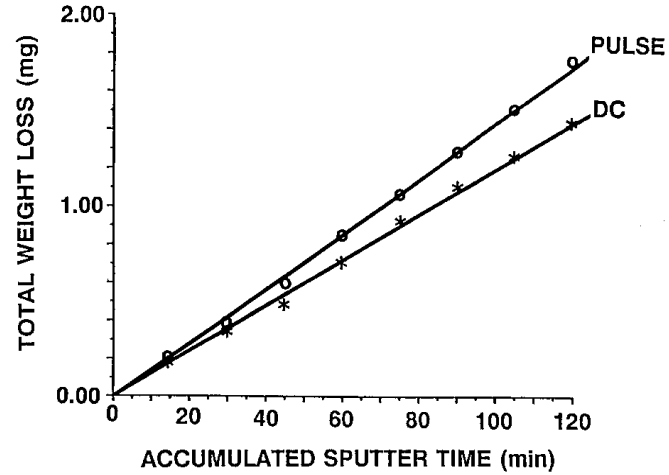

Figure 2. Comparison of the weight loss for a copper pin by using $\mathrm{dc}$ and pulsed glow discharges.

current. Figure 2 shows the weight loss for a copper pin at intervals over an accumulated time of $2 \mathrm{~h}$. The difference in the pulsed and dc modes, although not dramatic, is clearly evident. In general, an enhancement of about $10-15 \%$ is noted, an effect somewhat lower than the $40-45 \%$ enhancement from the sputter yield data shown in Figure 3 (adapted from Chapman [11]). It may be that the higher ion energies present in the pulsed discharge affect the observed sputter yield nearer the portion of the curve that has a smaller slope. The sputter yields in Figure 3, obtained under higher vacuum conditions, are not directly comparable with our glow discharges, but the qualitative trend is similar. Pulse frequencies of 20,60 , and $100 \mathrm{~Hz}$ at $50 \%$ duty cycles were investigated, and comparable differences in weight loss were observed in each case.

A comparison of the ion signals obtained from the pulsed and dc sources is shown in Figure 4. Average pulse discharge currents were compared to the same value of dc current. Compared to the sputter yield enhancement, the ion signal increase is much larger, by about a factor of 2 . The factors affecting ionization are much more numerous and complex than those for the simpler sputter ablation step. For example, during the "on" cycle, the higher voltage and higher current pulse create a plasma configuration that may more efficiently ionize analyte species.

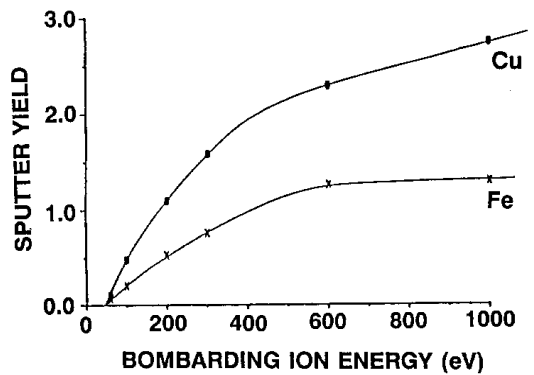

Figure 3. Sputter yields for copper and iron at various ion energies, as adapted from Chapman [11].

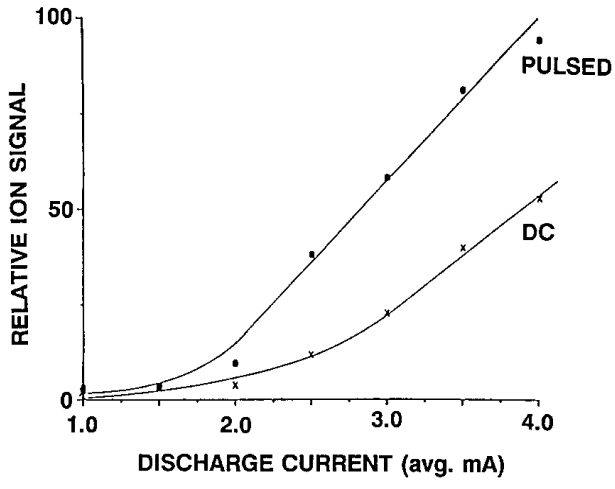

Figure 4. Ion signal dependence for a pulsed versus dc argon glow discharge at 1.0 torr. Sample was an SRM C1101 brass pin.

\section{Pulse Patterns}

During investigation of the effect of the pulse discharge, several anomalies were observed in the peak shapes of the gas and sputtered species. Instead of the anticipated square-wave pattern, reproducible peaks were observed at the beginning or end of the pulse, depending on the species under consideration. Figure 5 shows oscilloscope traces for the duration of one pulse cycle, along with the response of the power supply as measured at the cathode. The sudden increase in the sputtered species signal after termination of the pulse to form an "afterpeak" or "postpulse" (Figure 5b) was found to be typical for sample components, metal dimers, and metal argides. In contrast, only gaseous impurities show a "prepeak" (Figure 5c), a sudden rise in ion signal when the discharge is first turned on, followed by decay to a plateau value for the duration of the "on" duty cycle. A brief induction period of about $0.7 \mathrm{~ms}$ is observed in Figure $5 \mathrm{~b}$ before the appearance of ions arising from sputtered atoms. Following each pulse, the surface of the cathode reequilibrates with the gaseous environment. Reinitiation of the next pulse results in a stripping away of these surface layers by sputtering before the sample atoms begin to appear. For example, residual water vapor may cause oxide layers to form on the hot surface between pulses. The afterpeak appears about $0.15 \mathrm{~ms}$ after the high voltage terminates and is sustained for the next 2-3 ms before decaying to the baseline. Figure $5 \mathrm{c}$ illustrates the rapid rise of the prepeak (within $0.5 \mathrm{~ms}$ ), followed by a slow decay lasting about $2 \mathrm{~ms}$ before the plateau ion value is reached.

\section{Effect of Duty Cycle}

The intensity of the afterpeak varies with the applied voltage. If, at a fixed frequency, the duty cycle is reduced (e.g., from $50 \%$ to $25 \%$ ), the peak voltage and current must be increased to achieve the same number of ions arriving at the cathode surface during the pulse period. Figure 6 shows the effect on the rela- 


\section{POWER SUPPLY}

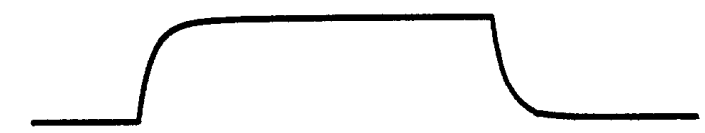

A
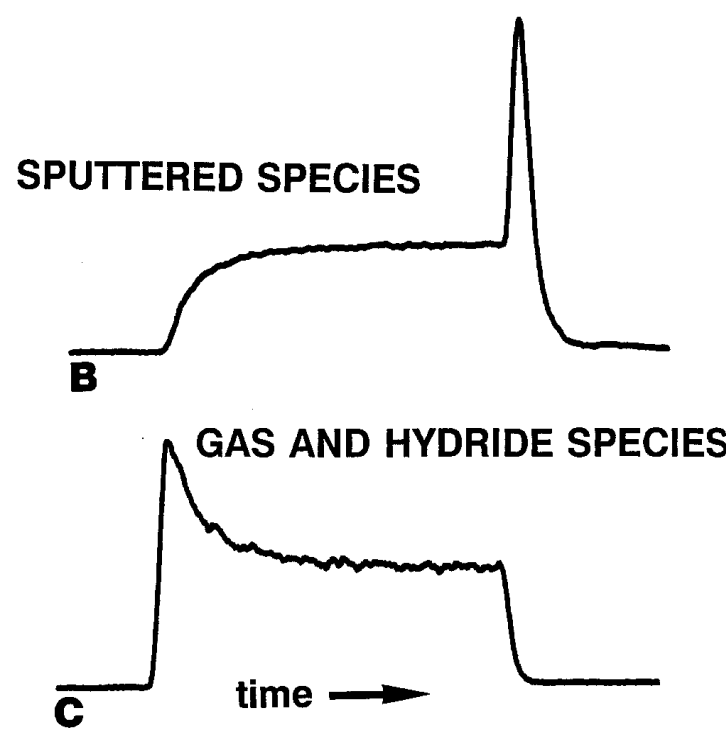

Figure 5. Single mass traces over the pulse period, $1000 \mathrm{~V}$ discharge at $50 \mathrm{~Hz}$ and $50 \%$ duty cycle. (a) Response of the power supply at the cathode, (b) typical sputtered species signal $\left({ }^{54} \mathrm{Fe}^{+}\right)$, (c) typical gas species signal $\left(\mathrm{H}_{3} \mathrm{O}^{+}\right)$. See text for explanation. tive afterpeak signal intensity as the duty cycle is varied from $25 \%$ to $75 \%$. The higher voltages required to maintain the constant current at the shorter duty cycles yield higher afterpeaks, a factor that suggests certain analytical advantages. Argon metastable atoms, which are responsible for a significant portion of the ioniza- tion in the discharge $[12,13]$, may also play a role in the formation of the sputtered species afterpeak. In a steady-state condition, as in the dc discharge, the metastable population is a balance of formation processes (collisions of argon atoms with secondary and thermal electrons conforming to a Boltzmann distri-
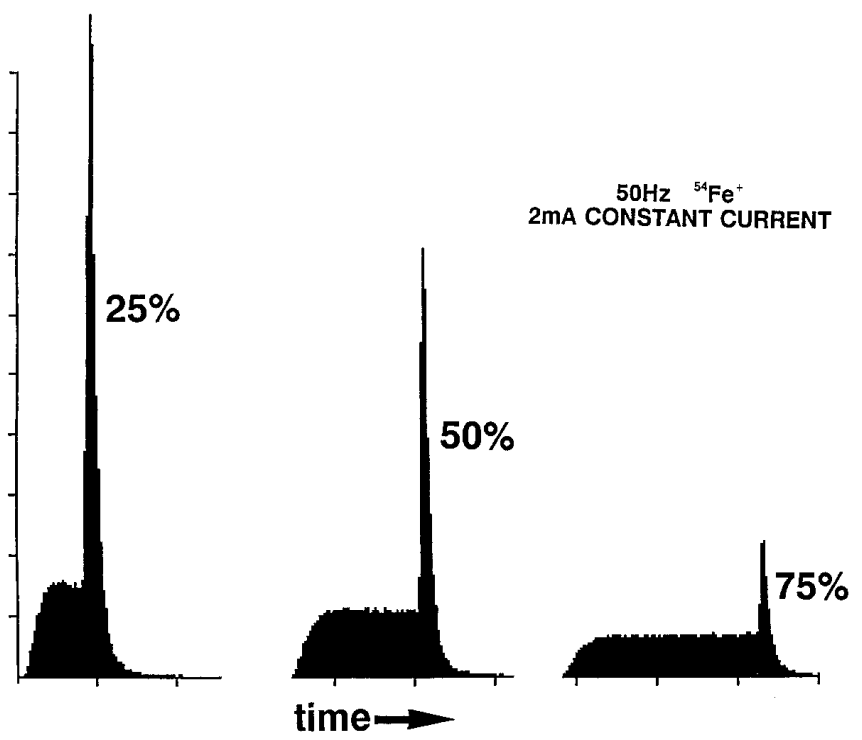

Figure 6. The effect of duty cycle on the size of the afterpeak. Applied voltage for $25 \%, 1800 \mathrm{~V} ; 50 \%$, $1075 \mathrm{~V} ; 75 \%, 850 \mathrm{~V}$. 


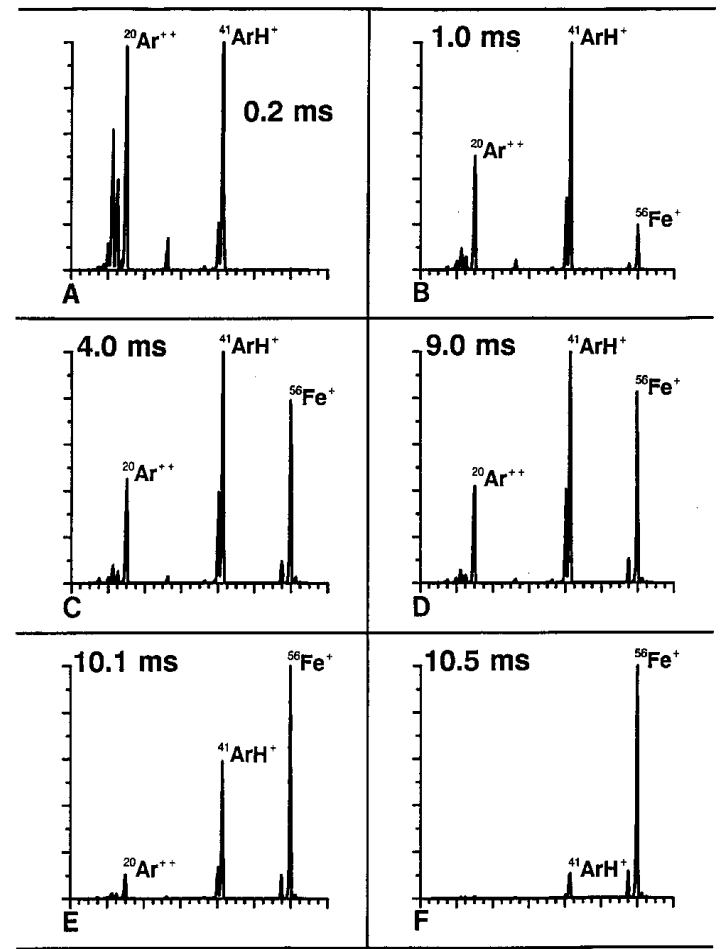

Figure 7. Time-resolved study over a $50 \%$ pulse duty cycle with a $0.3-\mathrm{ms}$ data gate and a pulse frequency of $50 \mathrm{~Hz}$. Indicated with each spectrum is the delay time at which the data gate opened after the start of the pulse. All spectra are normalized to the highest peak. From (b) to (f) there is an eightfold increase in the ${ }^{56} \mathrm{Fe}^{+}$intensity.

bution) and destruction processes (collisions with fast electrons, neutral atoms, other metastable species, and the vessel wall) [14]. Secondary electron emission from the cathode replenishes electrons lost in the plasma collisions and maintains the average electron temperature. When the applied potential is shut off and the electric field collapses, the sample surface is no longer bombarded by argon ions, so the source of secondary electrons is eliminated. Energetic electrons still in the glow region begin to lose their kinetic energy through collisions. As the average electron temperature decreases, more electrons can combine with positive argon ions to produce excited argon atoms, which are trapped in the metastable state or decay to groundstate atoms. The sudden increase in the metastable population could cause Penning ionization of the neutral sputtered species that are still randomly moving in the discharge space (or associatively ionize the neutral particles to form metal argides). Phelps and Molnar [15] discuss the repopulation of argon metastable particles in the dark space region of their spark discharge. Kartazaev et al. [16] observed similar phenomena in a helium spark discharge. We believe that the metastable formation plays a significant role in the afterpeak formation of the pulsed glow discharge.

\section{Time Resolution Studies}

The time-dependent phenomena evident in Figure 5 led us to explore more fully the ion profiles of the pulsed glow discharge mass spectrum by carefully placing a narrow data gate over different portions of the pulsed period. The profile of a $50-\mathrm{Hz}, 50 \%$ duty cycle pulsed discharge is illustrated in Figure 7. Each spectrum presents information collected through a 0.3$\mathrm{ms}$ data gate. The opening of the gate was delayed to enable sampling of different portions of the pulse period. Figure $7 \mathrm{a}$ indicates that as the discharge begins to come on, only argon and contaminant gas species (which are ionized by EI) can be seen in the mass spectrum. After approximately $1 \mathrm{~ms}$, the sputtered signals are detected, but the gas species still dominate the mass spectrum. It is not until the ion signals reach their plateau values (usually around 3-4 ms after the start of the pulse period) that an equilibrium is reached in the spectrum. In fact, a spectrum taken in the plateau region of the pulse period closely resembles a dc spectrum obtained at approximately the same voltage. At $50 \mathrm{~Hz}$ and a $50 \%$ duty cycle, the discharge is "on" for only $10 \mathrm{~ms}$. Figure $7 \mathrm{e}$ indicates that as the discharge begins to shut off, the gas species rapidly disappear, and the sputtered species signals become the sole feature of the mass spectrum. Because the mass spectrum would ideally be composed only of sample ions, data gates set over the afterpeak yield a cleaner analytical response.

\section{Spectral Discrimination}

An ability to separate the ion contribution of the background discharge gases from that of the sputtered species would be of great value, given the fact that spectral interferences arising from the gas components can cause serious problems in GDMS. Because the gas and sputtered species behave differently over the pulse period, our interest was to discriminate against one or the other by careful placement of the data acquisition window. One region that is particularly affected by gaseous interferences is the vicinity of the silicon isotopes, where such prominent species as $\mathrm{COH}^{+}$and $\mathrm{N}_{2} \mathrm{H}^{+}$are troublesome. Figure 8a shows a dc spectrum of an SRM 1261 pin that clearly demonstrates how the interfering gas ions can obscure the ion signal from the sputtered sample components. In contrast, Figure $8 \mathrm{~b}$ was acquired through a narrow data gate placed in the region shortly after the crest of the sputtered afterpeak and beyond the point where the gaseous ion signals had decayed. The use of the gated pulse method results in an isotope ratio for the silicon that is within $1 \%$ of accepted values.

The use of the pulsed glow discharge as a tool to discriminate against interfering species is just one application of this method. Studies are currently under way to analyze in depth the mechanisms that influence formation of both the prepeak and afterpeak. 


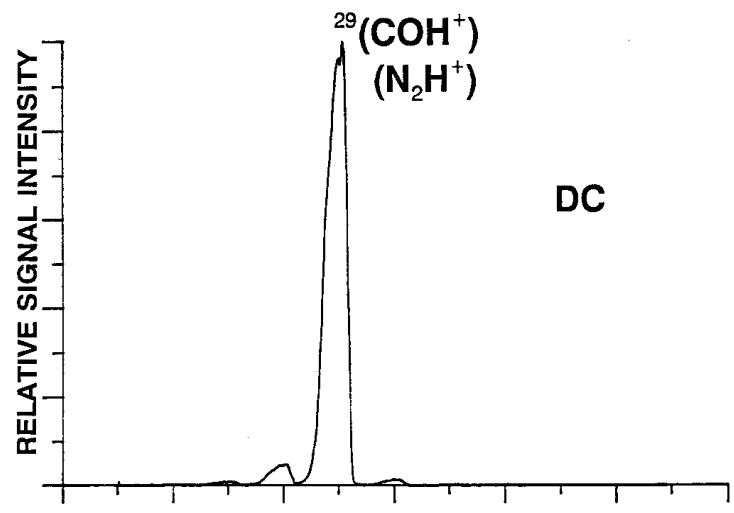

a

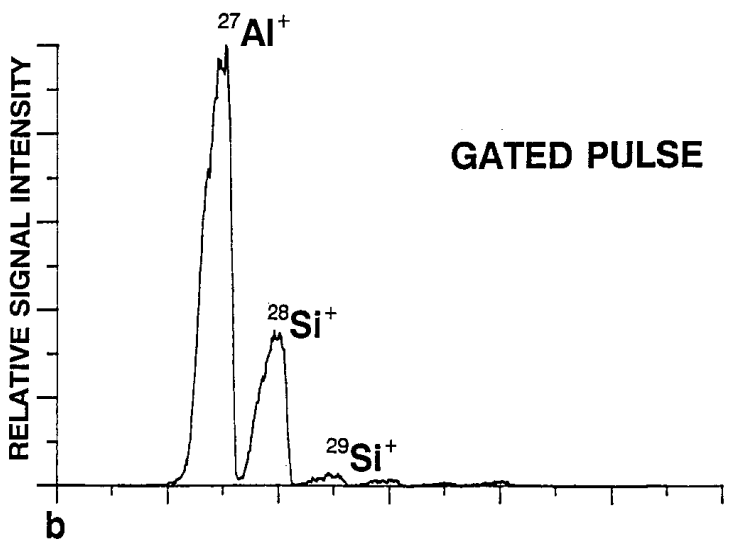

Figure 8. Mass spectra of an SRM 1261 pin. (a) A typical dc spectrum; (b) gated to allow only the ion signal from the afterpeak region to be sampled. Both spectra were obtained from a 3-mA average current discharge at a pressure of 1.3 torr of argon.
This includes the use of different waveforms (sawtooth waves, triangle waves, etc.), simultaneous use of multiple discharges, and spectroscopic analysis of metastable populations in the negative glow region. Understanding time-dependent properties of the pulsed discharge will lead to a better grasp of the fundamental factors that control glow discharge plasmas.

\section{Acknowledgment}

This work has been supported by a grant from the U.S. Department of Energy.

\section{References}

1. Harrison, W. W.; Bentz, B. L. Prog. Anal. Spectrom. 1988, 11, 53.

2. Loving, T. J.; Harrison, W. W. Anal. Chem. 1983, 54, 1523.

3. Cobine, C. D. Gaseous Conductors; Dover: New York, 1958; p 207.
4. Dawson, J. B.; Ellis, D. J. Spectrochim. Acta 1967, 23A, 565.

5. Barnett, W. B.; Kahn, H. L. Anal. Chem. 1972, 44, 935.

6. Mitchell, D. G.; Johansson, A. Spectrochim. Acta 1969, 25B, 175.

7. De Jong, G. J.; Piepmeier, E. H. Spectrochim. Acta 1974, 29B, 159.

8. Bruhn, C. G.; Bentz, B. C.; Harrison, W. W. Anal. Chem. 1978, 50, 373 .

9. Wehner, G. K. In Methods and Phenomena: Their Applications in Science and Technology; Wolsky, S. P.; Czanderna, A. W., Eds.; Elsevier: New York, 1975; Vol. 1, Chapter 1.

10. Davis, W. D.; Vanderslice, T. A. Phys. Rev. 1963, 131, 219.

11. Chapman, B. Glow Discharge Processes; Wiley: New York, 1980; p 394.

12. Coburn, J. W.; Kay, E. Appl. Phys. Lett. 1971, 18, 435.

13. Smyth, K. C.; Bentz, B. L.; Bruhn, C. G.; Harrison, W. W. J. Am. Chem. Soc. 1979, 101, 797.

14. Hardy, K. A.; Shelden, J. W. J. Appl. Phys., 1982, 53, 8532.

15. Phelps, A. V.; Molnar, J. P. Phys. Rev. 1953, 89, 1202.

16. Kartazaev, V. A.; Piotrovskii, Yu. A..; Tolmachew, Yu. A. Opt. Spektrosk. 1977, 42, 1048. 